\title{
Dynamic Data Driven Applications Systems: New Capabilities for Application Simulations and Measurements
}

\author{
Frederica Darema \\ National Science Foundation, Arlington VA 22230, USA \\ darema@nsf.gov
}

\begin{abstract}
The Dynamic Data Driven Application Systems (DDDAS) concept entails the ability to incorporate dynamically data into an executing application simulation, and in reverse, the ability of applications to dynamically steer measurement processes. Such dynamic data inputs can be acquired in real-time on-line or they can be archival data. DDDAS offers the promise of improving modeling methods, augmenting the analysis and prediction capabilities of application simulations, improving the efficiency of simulations and the effectiveness of measurement systems.

In the recent years grid computing technologies provide advanced computational capabilities for applications and application simulations At the same time measurement infrastructures, from instruments to sensor systems, to data storage technologies and remote data access have also matured. The DDDAS concept dynamically integrates computational and measurement aspects of an application, and in that respect the "platform" supporting the application becomes a unified computational and measurement infrastructure. Enabling the synergistic feedback and control-loop between application simulations and measurements requires novel application modeling approaches and interfaces to measurement systems, mathematical and statistical algorithms tolerant to perturbations from dynamic data inputs, and systems software to support the dynamic resource requirements of such applications. This and the rest of the papers in the proceedings of this workshop provide examples of ongoing research developing DDDAS technologies, both in terms of new capabilities in applications, measurement methods and in related systems software technologies.
\end{abstract}

\section{Introduction}

The Dynamic Data Driven Application Systems (DDDAS) [1,2] concept entails the ability to incorporate dynamically data into an executing application simulation, and in reverse, the ability of applications to dynamically steer measurement processes. Such dynamic data inputs can be acquired in real-time on-line or they can be archival data. The DDDAS concept offers the promise of improving modeling methods, augmenting the analysis and prediction capabilities of application simulations, improving the efficiency of simulations and the effectiveness of measurement systems. 
Traditional applications and systems simulations, thought fairly complex, are still lacking the ability to accurately describe such systems. This situation is accentuated in cases where real-time dynamic conditions exist. Application simulations that can dynamically incorporate at runtime new data, archival or from on-line measurements of the actual systems, offer the promise of more accurate analysis, more accurate predictions, more precise controls, and more reliable outcomes. These capabilities are fostered with the DDDAS concept. DDDAS can be viewed as a methodology to counterbalance incompleteness in model and capability to enhance the application models by imparting additional information into the model as at runtime additional data are used to selectively enhance or refine the original model. It can also be used to reduce the time to derive the simulation results, an important capability in many simulations, including simulations of real-time events.

In addition the ability of an application simulation to control and guide the measurement processes, creates more effective measurement processes. Such capabilities are highly desirable in cases where measurements are difficult to perform, time-critical, or expensive, in that can reduce collection time, cost, improve the quality of data collected.

Thus the concept of DDDAS goes beyond the current notions of "systems of systems" which primarily refer to multi-modal computational aspects of an application. DDDAS extends the concept of the "systems of systems' to also include the "measurement" as one of the systems.

In the recent years technologies to support the computational aspects of applications and application simulations have considerably advanced, as exemplified by the advent of grid computing technologies and computational grid infrastructures. At the same time measurement infrastructures, from instruments to sensor systems, to data storage technologies and remote data access have also matured. More systematic infrastructures for computing platforms and for measurement systems have been also recently called for [4]. The DDDAS concept dynamically integrates computational and measurement aspects of an application, and in that respect the "platform" supporting the application becomes the unified computational and measurement infrastructure. This kind of integrated, unified infrastructure goes beyond the current notions of computational grids; one could call it the "extended grid" or the integral grid.

To enable the DDDAS paradigm requires synergistic multidisciplinary research projects. Research in such projects needs to address in an integral way application modeling and simulation methods, mathematical and statistical algorithms, measurement methods, and systems software to support DDDAS environments. Opportunities in enabling DDDAS and challenges in creating DDDAS capabilities, have been discussed in [1,2,3] and elsewhere. A brief list of such challenges includes: advances at the applications level for enabling this kind of dynamic feedback and coupling with measurements; advances in complex, multi-modal models, and ability to combine dynamically such models as is dictated by the injected data, advances in the applications algorithms for the algorithms to be amenable to perturbations by the dynamic data inputs and enhanced capabilities for handling uncertainties in input data; new technology in the computer software systems areas to support such environments where the applications requirements are dynamic, depending on the dynamic data inputs. Furthermore the DDDAS concept by integrating the computational and measurement aspects of an application, goes beyond the notions of 
cyber-infrastructure. Thus many aspects such as reliability, security, fault tolerance, system recovery extend beyond the technologies developed for computational grids, to accommodate the support not only of computational grids but also of systems like sensor networks and other measurement instruments.

The efforts presented here provide examples of technologies and capabilities that are being developed based on the DDDAS concept and supporting DDDAS environments. This workshop is a third of a series of workshops of the DDDAS topic that have been organized as part of ICCS, starting with ICCS'03.

\section{Overview of Selected Research Efforts Presented in This Workshop}

The rest of the papers presented in this workshop represent ongoing research projects each addressing a number of aspects along the technical challenges mentioned above. With the exception of project [16], all other projects [6-15, 17-19] are predominantly funded by NSF ITR program [5]. Several papers address application simulations whose capabilities are enhanced by dynamic data inputs into the executing application/simulation. While many of the papers are developing new capabilities at the application and algorithm levels, several of the papers focus in the underlying software technology to support the development of the complex DDDAS applications, their dynamic runtime support requirements and interfaces to control measurement systems. Several papers provide examples of simulation controlled measurement processes. Most of the work on mathematical algorithms tolerant and stable to dynamically streamed data is done in the context and together with the development of the application models. In all cases the mathematical algorithms and systems software development is done in the context of specific applications driving and validating the developed technologies.

The projects discussed in [6-11,21] enable improved analysis and prediction capabilities of application simulations and/or speeding up the computation times together with improved quality of simulations, and in a feedback loop the simulations control of measurements, sensors and other instruments. The applications range from structural analysis, environmental and natural resource management, and crisismanagement. In [6] the project employs sensor originating data-driven pre-computed solutions synthesis to speedup simulation time and fidelity of result for applications that have real-time constraints such as supersonic platforms and fire dynamics simulations. In [7] employs dense networks of high resolution phased array and doppler radars to adaptively respond to weather patterns and produce better-than-realtime predictions of tornadoes and other "meso-scale" weather events by simulations and measurements continually steering by new data, optimizing data collection, steering remote observing and selectively changing configuration of measurement instruments. The paper in [8] develops capabilities for short-range forecasting of wildfire behavior from real-time weather data, thermal imaging, and thermal sensor data streams. In [9] algorithms to incorporate measurements in algorithms for underground contaminant simulations, and in [10] efficient algorithms to dynamically integrate observational data with models for improved forward and backward (adjoint) atmospheric chemical transport modeling. The project in [11] discusses 
development of application models and algorithms, as well as a systems software infrastructure for optimization of the process of secondary oil recovery. The project in [21] has developed high-fidelity simulations of large structures, subject to mechanical and thermal stesses.

Applications in [12-14] are in the areas of medical and biological sciences. The project in [12] uses the DDDAS concept to enable real-time intra-operative image guided neurosurgery, by developing applications and the underlying infrastructure to support such environments. In [13] spectroscopic data are dynamically input into protein dynamics computations speeding-up and improving the fidelity of the computations. [14] develops models to simulate the air flow around bat wings during flight.

The projects in [15-17] develop multimodal simulation models and integration of such multimodal models. The project in [15] develops simulations for decision support systems, such as case scenario for evacuation of a building in case of fire and structural damage to a building due to an explosion. In such "system of systems" simulation scenario, numerical PDE and FEM models simulating fire dynamics and structural aspects of the building are integrated $\mathrm{w}$ agent based models that simulate behavior of people in a panic situation, evacuation policies, and other factors. The models are coupled and also driven by dynamic data inputs from sensors monitoring smoke and temperature increases due to the fire, sensors monitoring air flow, and feeding all such data into the fire model, to other sensors monitoring structural integrity aspects, and feeding that to the structural analysis program, and in turn such models coupled with the agent based evacuation simulation models. Under the same project simulations are developed when not only a building but a section of a city is affected. The project in [16] is using the DDDAS concept, to develop a decision support system for emergency medical services. One can envision the scenario, where the capabilities in [15] are further coupled with those in [16] to develop an end-to-end decision support system of how to evacuate and optimize medical services in case of an adverse attack to a building or a city section. Projects [15] and [16] coupled with [21,] which is simulating the crash of an aircraft onto a building, could be used to fully simulate the scenario of a crisis situation caused by such an adverse event. The project in [17] uses the DDDAS concept to couple continuous and discrete modeling methods for 3D tracking, and overcoming the shortcomings of each of these two methods when applied individually. Improvements in such methods can enable computer-based sign-language analysis, and other human-computer interaction applications such, human-identification and computer-based recognition of human deception.

The projects in [18-20] address systems issues and other fundamental aspects of DDDAS systems, such as: ability to invoke dynamically application modalities as dictated by the dynamic data inputs [18], runtime environments to support the faulttolerant underlying infrastructure required by DDDAS systems [19], and aspects of how such "systems-of-systems" environments, where dynamic multimodal models and measurement systems are integrated can be validated.

\section{Summary}

The DDDAS concept opens new capabilities in applications and measurements. Through efforts that have started developing DDDAS systems, that promise begins to 
materialize. While we are far from having fully and robustly developed these capabilities and the underlying technologies, we are in a steady path towards accomplishing that objective. The path is long, and the progress made is exemplified by the projects presented in the ICCS/DDDAS workshops, including the present one.

\section{References}

1. NSF Workshop, March 2000; www.cise.nsf.gov/dddas

2. F. Darema, Dynamic Data Driven Applications Systems: A New Paradigm for Application Simulations and Measurements, ICCS'04

3. F. Darema, Grid Computing and Beyond: The Context of Dynamic Data Driven Applications Systems, Proceedings of the IEEE, Special Issue on Grid Computing (in $3 / 05)$

4. Cyberinfrastructure Report http://www.communitytechnology.org/nsf_ci_report

5. NSF Information Technology Research (ITR) Program (1999-2004)

6. J. Michopoulos, C. Farhat, E. Houstis, P. Tsompanopoulou, H. Zhang, and T. Gullaud, Dynamic Data Driven Methodologies for Multiphysics System Modeling and Simulation; Proceedings ICCS'05

7. Beth Plale, Dennis Gannon, Dan Reed, Sara Graves, Kelvin Droegemeier, Bob Wilhelmson, and Mohan Ramamurthy, Towards Dynamically Adaptive Weather Analysis and Forecasting in EAD; Proceedings ICCS'05

8. Jan Mandel, Lynn S. Bennethum, Mingshi Chen, Leopoldo P. Franca, Craig J. Johns, Janice L. Coen, Craig C. Douglas, Minjeong Kim, Andrew V. Knyazev, Robert Kremens, Vaibhav Kulkarni, Guan Qin, Anthony Vodacek, Jianjia Wu, Wei Zhao and Adam Zornes, Towards a Dynamic Data Driven Application System for Wildfire Simulation; Proceedings ICCS'05

9. Craig C. Douglas, Yalchin Efendiev, Richard Ewing, Victor Ginting, Raytcho Lazarov, Martin J. Cole, Greg Jones and Chris R. Johnson, Multiscale Interpolation, Backward in Time Error Analysis for Data-Driven Contaminant Simulation; Proceedings ICCS'05

10. Adrian Sandu, Emil M. Constantinescu, Wenyuan Liao, Gregory R. Carmichael, Tianfeng Chai, John H. Seinfeld, and Dacian Daescu, Ensemble-Based Data Assimilation for Atmospheric Chemical Transport Models; Proceedings ICCS'05

11. Manish Parashar, Vincent Matossian, Wofgang Bangerth, Hector Klie, Benjamin Rutt, Tahsin Kurc, Umit Catalyurek, Joel Saltz, and Mary F. Wheeler, Towards Dynamic DataDriven Optimization of Oil well Placement; Proceedings ICCS'05

12. Amit Majumdar, Adam Birnbaum, Dong Ju Choi, Abhishek Trivedi, Simon K. Warfield, Kim Baldridge, and Petr Krysl, A Dynamic Data Driven Grid System for Intra-operative Image Guided Neurosurgery; Proceedings ICCS'05

13. Dimitrios Morikis, Christodoulos A. Floudas, and John D. Lambris, Structure-based Integrative Computation and Experimental Approach for the Optimization of Drug Design; Proceedings ICCS'05

14. I.V. Pivkin, E. Hueso, R. Weinstein, D. H. Laidlaw, S. Swartz, and G. E. Karniadakis, Simulation and Visualization of Air Flow Around Bat Wings During Flight; Proceedings ICCS'05

15. R. Chaturvedi, S. A. Filatyev, J.P. Gore, A. Hanna, J. Means, and A.K. Mellema, Integrating Fire, Structure and Agent Models; Proceedings ICCS'05

16. Mark Gaynor, Margo Seltzer, Steve Moulton, and Jim Freedman,, A Dynamic, DataDriven, Decision Support System for Emergency Medical Services; Proceedings ICCS'05 
17. Dimitris Metaxas and Gabriel Tsechpenakis, Dynamic data Driven Coupling of Continuous and Discrete Methods in 3D Tracking; Proceedings ICCS'05

18. David Brogan, Paul Reynolds, Robert Bartholet, Joseph Carnahan, and Yannick Loitiere, Semi-Automated Simulation Transformation for DDDAS; Proceedings ICCS'05

19. Andrew Grimsahw, Marty Humphrey, John C. Knight, Anh Nguyen-Tuong, Jonathan Rowanhill, Glenn Wasson, and Jim Basney, The Development of Dependable and Survivable Grids; Proceedings ICCS'05

20. J. Michopoulos and Sam Lambrakos, On the Fundamental Tautology of Validating Data-Driven Models and Simulations; Proceedings ICCS'05

21. Christoph Hoffman, Ahmed Sameh, Ananth Grama, High-Fidelity Simulation of Large Scale Structures; Proceedings ICCS'05 